\title{
Clinician-Focused Overview and Developments in Polysomnography
}

\author{
Leslie C. Markun ${ }^{1} \cdot$ Ajay Sampat ${ }^{1}$ \\ Accepted: 9 November 2020 / Published online: 23 November 2020 \\ (C) Springer Nature Switzerland AG 2020
}

\begin{abstract}
Purpose of Review Polysomnography (PSG) represents a fundamental diagnostic tool used in the evaluation of sleep disorders. It represents a simultaneous recording of sleep staging, eye movements, electromyographic tone, respiratory parameters, and electrocardiogram. It is particularly helpful in the assessment of sleep-disordered breathing and its management, propensity for excessive sleepiness, complex behaviors during sleep, including motor disturbances of sleep, sleep-related epilepsy, and parasomnias. This review is intended to summarize the indications for PSG, the limitations and challenges of this diagnostic tool, indications for home sleep apnea testing options, and new developments and trends in polysomnography.

Recent Findings The polysomnogram is fundamentally important in the evaluation of sleep-disordered breathing in the setting of cardiovascular comorbidities and neurologic conditions such as neuromuscular disease, stroke, and epilepsy and in the evaluation of dream enactment behavior in the setting of REM sleep behavior disorder (RBD). Because RBD is predictive of neurodegenerative disorders, recent data highlights the importance of PSG in corroborating the diagnosis of RBD and identifying people who may be at risk. However, due to cost as well as limitations in access to care, further testing has been developed and implemented including the home sleep apnea test (HSAT). The evolution of consumer wearable devices has also been a growing trend in sleep medicine; however, few have received appropriate validation.

Summary PSG has been used in both the clinical and research settings and remains the gold standard clinical diagnostic test for suspected obstructive sleep apnea (OSA) or central sleep apnea (CSA). Clinicians must be familiar with the basic indications for a PSG but also recognize when it is absolutely required. At this time, the PSG is essential in the evaluation of nocturnal hypoventilation disorders of sleep, periodic limb movements of sleep, and central nervous system hypersomnia (in the absence of CSF hypocretin) when combined with the multiple sleep latency test (MSLT) and is probably the only way to help differentiate among complex behaviors during sleep, especially in the setting of RBD. The capacity to establish an early diagnostic risk of potential dementia would be of critical importance once neuroprotective agents become available.
\end{abstract}

Keywords Polysomnography $\cdot \mathrm{PSG} \cdot$ Sleep study $\cdot$ Home sleep apnea testing $\cdot$ Multiple sleep maintenance test $\cdot$ EMG montage . EEG montage

\section{Introduction}

Because of the limitations of subjective measures of sleep, there is often an inherent need for an objective measure to

This article is part of the Topical Collection on Sleep and Neurological Conditions

Ajay Sampat

acsampat@ucdavis.edu

Leslie C. Markun

lmarkun@ucdavis.edu

1 Division of Sleep Medicine, UC Davis Department of Neurology, 4860 Y Street, Suite 3700, Sacramento, CA 95817, USA evaluate sleep quality and disorders which impair sleep. Polysomnography (PSG) is defined as the continuous monitoring and simultaneous recording of physiologic activity during sleep [1]. Hans Berger invented electroencephalography (EEG) in the early twentieth century which records electrical cortical activity and calculates relative differences in electrical fields across brain regions. Thereafter, clinicians integrated this technology and incorporated additional physiologic respiratory and cardiac parameters to develop the sleep study [2]. The term polysomnogram was coined in the 1970s and combined continuous EEG, which analyzed sleep states, with respiratory channels, body position sensors, and electrocardiography (ECG). Initially, analog (paper) recordings had limitations in the selected EEG montage and numbers of possible channels, and the digitization in the 1990s allowed for many 
more parameters to be analyzed. In recent years, additional parameters were incorporated including electromyography (EMG) tone, electrooculogram (EOG), expanded EEG montages, and transcutaneous or end-tidal capnography. PSG has become the gold standard for comprehensive monitoring of sleep to evaluate sleep disorders. In addition, video recording has led to video PSG (VPSG), which is a standard attribute in the evaluation and assessment of complex behaviors during sleep allowing the capacity to record movements, speech, behaviors including assessment for amnesia, recovery, and patients' response to questions by the sleep technicians [3].

In order to standardize PSG across sleep labs, the American Academy of Sleep Medicine (AASM) Manual for Scoring of Sleep and Associated Events was developed to provide the universally accepted criteria for standard recording technique and scoring guidelines (AASM Scoring Manual version 2.6, 4). Sleep is staged in 30-s epochs with detailed criteria and definitions for wakefulness and each stage of sleep. EEG is recorded at the frontal, central, and occipital regions and is used to define sleep stages [4]. EOG and chin EMG help with the evaluation of the sleep stage, particularly during rapid eye movement (REM) sleep. Airflow via nasal pressure sensors detects partial airflow limitation or hypopneas, and airflow via oronasal thermal flow sensors detects complete airflow obstruction or apneas. To further classify apneas as obstructive, mixed or central chest and abdominal sensors are used to evaluate respiratory effort [5]. Pulse oximetry detects the arterial oxygen saturation, and ECG detects the cardiac rate and rhythm. The anterior tibial EMG leads can detect periodic limb movements in sleep. End-tidal $\mathrm{PCO}_{2}$ can assist with the detection of hypoventilation [6]. Tables 1 and 2 provide a summary of the standard channels that are universally present in PSG and additional channels which can provide supplemental information for specific disorders (Fig. 1).

\section{Indications for Polysomnography}

Through systemic reviews and expert task force recommendation, the AASM release practice parameters for the indications for PSG and the strength of the available evidence $[7 \cdot \bullet$ ]. PSG is most commonly used to evaluate for and quantify the severity of sleep-related breathing disorders (SRBD) and titration of positive airway pressure in the treatment of SRBD. However, PSG can also be utilized in the evaluation of other sleep conditions, such as those of central nervous system hypersomnias (i.e., narcolepsy and idiopathic hypersomnia) and parasomnias (specifically REM behavior disorder), and in the assessment of periodic limb movements during sleep [1]. Though it is not required to diagnose all sleep conditions, such as insomnia and circadian rhythm disorders, PSG can serve as an essential diagnostic tool in certain cases where clinical history alone is not sufficient to make a diagnosis.

Table 1 Standard PSG channels

\begin{tabular}{|c|c|}
\hline Channel & Function \\
\hline $\begin{array}{l}\text { EEG (recommended are F4-M1, C4-M1, and O2-M1; at minimum } \\
3 \text { channels are required to stage sleep) }\end{array}$ & Monitoring of sleep staging, arousals, and abnormal or epileptiform activity \\
\hline $\mathrm{EOG} \times 2($ recommended are E1-M2 and E2-M2) & $\begin{array}{l}\text { Monitoring of horizontal and vertical eye movements to demonstrate in-phase and } \\
\text { out-of-phase deflections, which assist in sleep staging; electrodes are placed at the } \\
\text { right and left outer canthi, } 1 \mathrm{~cm} \text { above and below the eye axis }\end{array}$ \\
\hline $\begin{array}{l}\text { EMG (at minimum, } 3 \text { electrodes are required, typically } 3 \text { chin } \\
\text { electrodes, and additional } 2 \text { on anterior tibialis muscles) }\end{array}$ & $\begin{array}{l}\text { Evaluation of muscle tone to assist with sleep staging, RSWA (REM sleep without } \\
\text { atonia), and PLMS (periodic limb movements of sleep) }\end{array}$ \\
\hline ECG (single modified lead II placed on the torso) & Evaluation of heart rhythm to assess for arrhythmias and heart rate variability \\
\hline $\begin{array}{l}\text { Airflow (recommended are an oronasal thermal airflow sensor, } \\
\text { along with nasal pressure transducer) }\end{array}$ & $\begin{array}{l}\text { Detection of hypopneas and apneas. Differentiation between obstructive, central, } \\
\text { and mixed events }\end{array}$ \\
\hline Respiratory effort (abdominal and thoracic respiratory belts) & $\begin{array}{l}\text { Detection of respiratory effort to aid in the distinction between obstructive and } \\
\text { central respiratory events }\end{array}$ \\
\hline Pulse oximetry & $\begin{array}{l}\text { Detection of oxygen desaturation with respiratory events or due to pulmonary } \\
\text { disease }\end{array}$ \\
\hline $\begin{array}{l}\text { Body position sensor (optional parameter however typically } \\
\text { included) }\end{array}$ & $\begin{array}{l}\text { Evaluation of body position (via position sensors or by a technologist) to help } \\
\text { identify the positional component of sleep-disordered breathing }\end{array}$ \\
\hline Video monitoring & $\begin{array}{l}\text { Detection of unusual nocturnal behavioral events, including parasomnias, } \\
\text { movement disorders, and seizures }\end{array}$ \\
\hline
\end{tabular}

Standard PSG channels require at least 4 neurophysiology channels (EEG, EOG $\times 2$, EMG) and typically include ECG, airflow, pulse oximetry, and body position channels as well

$E E G$ electroencephalography, EOG electrooculogram, $E M G$ electromyography, $E C G$ electrocardiography, $R E M$ rapid eye movement, $P L M S$ periodic limb movements of sleep 
Table 2 Additional PSG channels

\begin{tabular}{|c|c|}
\hline Channel & Function \\
\hline $\begin{array}{l}\text { Expanded EEG (typically with } 8-10 \text { additional channels, } \\
\text { involving frontal, temporal, parietal, and occipital lobes) }\end{array}$ & Evaluation of ictal pattern, origin, and detection of sleep-related epilepsy \\
\hline $\begin{array}{l}\text { Expanded EMG (extensor digitorum muscles and additional } \\
\text { electrode over masseter muscle) }\end{array}$ & $\begin{array}{l}\text { Detection of PLMS, evaluation of motor tone in the setting of parasomnias } \\
\text { (particularly RSWA in RBD), and detection of bruxism }\end{array}$ \\
\hline Esophageal pressure monitoring (Pes) & $\begin{array}{l}\text { For evaluation of upper airway resistance syndrome. Provides a reflection of } \\
\text { intrathoracic pressure fluctuation associated with breathing efforts }\end{array}$ \\
\hline Nasal cannula pressure monitoring & $\begin{array}{l}\text { Detection of increased respiratory efforts by its effects on the inspiratory airflow } \\
\text { wave contour }\end{array}$ \\
\hline Nocturnal penile tumescence & Assessment of erectile dysfunction \\
\hline $\mathrm{CO}_{2}$ monitoring & $\begin{array}{l}\text { Using either transcutaneous or end-tidal } \mathrm{CO}_{2} \text {. For assessment of sleep-related } \\
\text { hypoventilation in obesity hypoventilation syndrome, for evaluation of } \\
\text { sleep-related breathing disorders in pediatric patients }\end{array}$ \\
\hline
\end{tabular}

Additional PSG channels can be added based on clinical indication

$E E G$ electroencephalography, EMG electromyography, RBD REM sleep behavior disorder, PLMS periodic limb movements of sleep. RSWA REM sleep without atonia

\section{The Utility of Polysomnography in the Assessment of Excessive Daytime Sleepiness}

A chief complaint of "excessive daytime sleepiness" (EDS) can be non-specific; thus, the first step in the evaluation of EDS is to obtain a detailed clinical history, particularly with a sleep diary to assess sleep duration and sleep regularity. The clinician should focus on sleep duration, sleep-wake patterns, and comorbid psychiatric and medical conditions, as well as any medications which can be temporally correlated to the onset of EDS. It is equally important to differentiate between bona fide sleepiness (which is defined as an increased propensity to fall asleep) and fatigue, which is a subjective sense of low energy and the need to rest, not relieved by sleep, and is more non-specific and can be seen in a variety of medical and

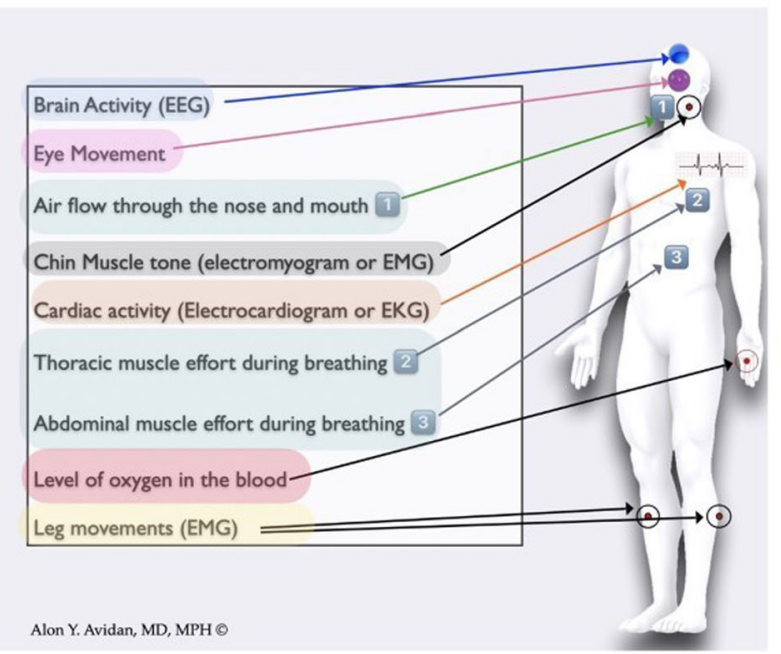

\begin{tabular}{l} 
LOC \\
ROC \\
\hline Fa \\
C \\
\hline L \\
\hline R \\
\hline St \\
\hline PTAFE \\
A \\
\hline C \\
\hline A \\
\hline S \\
(S)
\end{tabular}

Fig. 1 A standard PSG configuration consists of electroencephalography (EEG, measuring brain activity), electrooculography (EOG, measuring eye movements to assist in sleep staging), electromyography (EMG, measuring muscle tone in chin and limbs), electrocardiography (EKG, psychiatric conditions. Subjective questionnaires can be used to differentiate these symptoms and can help in the formulation of one's differential diagnosis. The most widely used questionnaire is the Epworth Sleepiness Scale (ESS), which is an 8-item inventory which assesses how likely subjects are to fall asleep or doze off during the day in common situations, each being rated on a 3-point scale. The total ESS score ranges from 0 to 24, with higher scores reflecting a greater tendency for sleepiness [8].

\section{Assessment of Sleep Apnea}

Historical elements are most suggestive of sleep-related breathing disorders (SRBD) including witnessed apneas, snoring, weight gain, hypersomnia especially on awakening,

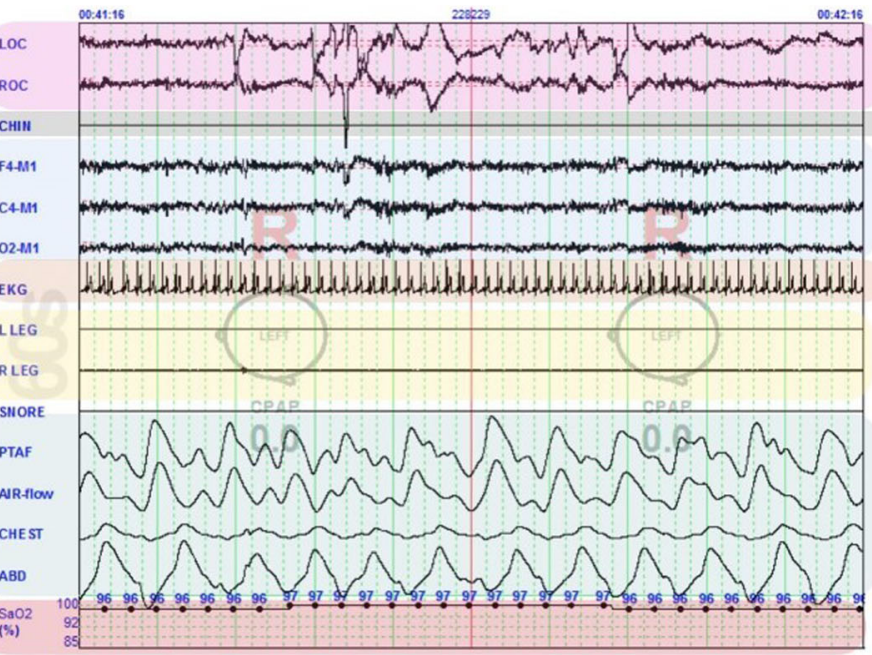

measuring cardiac activity), and respiratory channels (depicting airflow and effort) with pulse oximetry. These latter channels (respiratory and pulse oximetry) are most helpful in assessing for sleep-disordered breathing 
Table 3 STOP-BANG score for OSA

\begin{tabular}{lll}
\hline & 0 points & 1 point \\
\hline Do you snore loudly? & No & Yes \\
Do you often feel tired, fatigued, or sleepy during the daytime? & No & Yes \\
Has anyone observed you stop breathing during sleep? & No & Yes \\
Do you have (or are you being treated for) high blood pressure? & No & Yes \\
BMI & $\leq 35 \mathrm{~kg} / \mathrm{m}^{2}$ & $>35 \mathrm{~kg} / \mathrm{m}^{2}$ \\
Age & $\leq 50$ years & $>50$ years \\
Neck circumference & $\leq 40 \mathrm{~cm}$ & $>40 \mathrm{~cm}$ \\
Gender & Female & Male \\
\hline
\end{tabular}

STOP-BANG score to risk-stratify probability of having OSA. A score of $<3$ indicates a low risk of OSA, while a score of $\geq 3$ indicates a high risk of OSA morning headaches, or dry mouth, in addition to exam elements of elevated BMI, presence of hypertension, and signs of upper airway compromise [9]. Various questionnaires have been developed to help risk-stratify patients' likelihood of having obstructive sleep apnea (OSA), with the most commonly used being the STOP-BANG score [10]. This scale assesses common symptoms of OSA (snoring, witnessed apneas, and daytime fatigue) along with other patient medical comorbidities (hypertension) and clinical variables (BMI, age, neck circumference, and gender) and assigns a point for each of these elements (see Table 3 for full scale). A STOP-BANG score of $\geq 3$ indicates a high risk of OSA.

If OSA is suspected, then a sleep study (either PSG or home sleep apnea test (HSAT)) is indicated. The decision to proceed with PSG rather than HSAT is based on a patient's medical comorbidities, along with logistical considerations. In particular, patients, who have cardiorespiratory disease, potential respiratory muscle weakness due to a neuromuscular condition, hypoventilation or suspicion of sleep-related hypoventilation, chronic opioid medication use, and history of stroke, are recommended to obtain a PSG as HSAT has not been adequately validated in these populations and may underestimate or fail to diagnose a sleep disorder [7••]. HSAT is also more controversial in elderly populations especially when there is variation in probability to have significant OSA $[11,12]$. A HSAT may be more appropriate in patients who have a higher pretest probability of OSA without the previously listed comorbidities. Table 4 provides a list of common considerations when deciding between HST and PSG.

The apnea-hypopnea index (AHI) remains a primary electrographic criterion for the diagnosis of OSA, in a patient with the previously listed clinical symptoms, with further studies noting the oxygen desaturation index (ODI) as an important marker for cardiovascular health benefit given it can reflect the apnea depth and duration of apneas [13]. If there is high clinical suspicion of OSA, then a split-night diagnostic protocol can be ordered, in which a continuous positive airway pressure (CPAP) or bilevel positive airway pressure (BPAP) device is initiated overnight during the study to find appropriate positive pressure settings for treatment. A titration study is appropriate when a patient with previously diagnosed OSA requires formal testing to determine optimal pressure settings for treatment. Figure 2 demonstrates an example of a PSG from a patient with severe OSA, with frequent and

Table 4 Use of HST versus PSG

\begin{tabular}{ll}
\hline HST acceptable & PSG recommended \\
\hline High probability of OSA & History of congestive heart failure \\
Absence of comorbidities warranting PSG & Neuromuscular weakness \\
Delay in obtaining PSG & Suspicion of hypoventilation \\
Immobility or safety issues obtaining PSG & Use of chronic opioid medications \\
Strong "first-night effect" in a new sleep & Suspicion of parasomnia \\
environment & Suspicion of central sleep apnea \\
Absent indication for obtaining PSG & History of stroke (especially if recent) \\
& Low probability of OSA \\
& Previously non-diagnostic HST with high clinical suspicion of \\
& OSA \\
& Evaluation of central disorders of hypersomnolence (along with the \\
& MSLT) \\
& Evaluation of periodic limb movements in the setting of restless \\
& legs syndrome (RLS) \\
\hline
\end{tabular}

Comparison of when to obtain HST vs. PSG based on common clinical and logistical considerations 


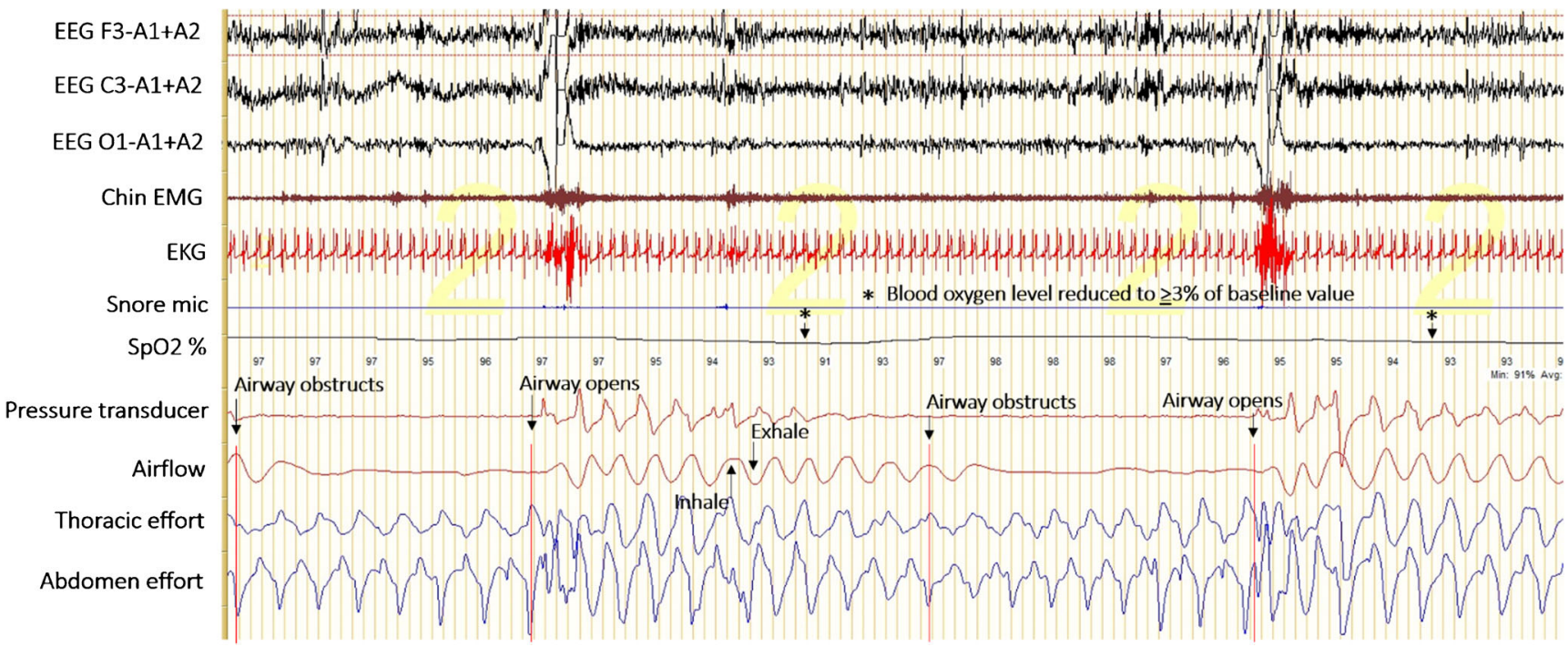

Fig. 2 Example of PSG in OSA. PSG demonstrating two obstructive apneic events as significantly reduced airflow with preserved respiratory effort, associated with severe desaturations during N2 sleep. Note the airway obstruction and airway opening with delayed blood oxygen level reduction. These PSG epochs included the following channels: EEG F3-A1+A2 left frontal in referential montage, EEG C3-

cyclic episodes of airflow limitation, accompanied by desaturation events.

\section{Assessment of Suspected Narcolepsy}

Polysomnography is also used as part of the diagnostic workup in suspected cases of central disorders of hypersomnolence, such as narcolepsy. Patients with suspected narcolepsy can have symptoms of excessive sleepiness with additional features, such as fragmented or poor nocturnal sleep, and REM intrusion phenomena, such as cataplexy, sleep-related hallucinations, or sleep paralysis. Cataplexy, in particular, is highly specific for narcolepsy and is defined as a sudden loss of muscle tone while a person is awake, leading to weakness and loss of voluntary muscle tone. This phenomenon is often triggered by sudden and strong positive emotions, such as laughter, excitement, or stress. In this clinical scenario, further evaluation is warranted with a nocturnal PSG followed by the daytime multiple sleep latency test (MSLT). Prior to obtaining these tests, a regular sleep pattern consisting of at least $7-8 \mathrm{~h}$ of sleep should be established, and any REM-suppressing medications and stimulants should be ideally held for at least 2 weeks (for $5 \times t^{1} / 2$ of drug with the longest active metabolite). If another sleep disorder such as OSA is detected, it must be treated prior to PSG and MSLT [6]. The following are criteria for both narcolepsy and idiopathic hypersomnia (IH), two of the most common disorders of central hypersomnolence, summarized from the International Classification of Sleep Disorders, volume 3 [14].
$\mathrm{A} 1+\mathrm{A} 2$ left central in referential montage, and EEG O1-A1+A2 left occipital in referential montage. Chin EMG, chin electromyography; EKG, electrocardiogram; snore mic, snoring sensor/microphone; $\mathrm{SpO}_{2} \%$, oxygen saturation; pressure transducer, nasal airflow; thoracic effort, thoracic/chest effort channel; abdomen effort, abdominal effort channel

A crucial component of the PSG which helps to depict an individual's sleep is the hypnogram. A hypnogram is a graph that represents the stages of sleep as a function of time (hours of sleep) and can serve as a visual summary of the brain wave activity from an electroencephalogram. The sleep stages that are typically recorded on the $y$-axis are the following: $\mathrm{W}$ (wake), R (REM sleep), N1 (stage N1 sleep or "light sleep"), N2 (stage N2 sleep or "deeper sleep"), and N3 (stage N3 sleep or slow-wave sleep). A normal hypnogram depicts non-REM predominant sleep (stages N1, N2, and N3) in the first half of the night, while REM sleep (stage R) becomes more predominant in the second half of the night. Stage R sleep is typically not observed before $90 \mathrm{~min}$ after sleep onset in a normal individual. However, in disorders of central hypersomnolence, both sleep-onset latency and REM onset latency are reduced, which can be visualized on a hypnogram. Specifically, for narcolepsy and idiopathic hypersomnia, a sleep-onset REM (SOREM) period within 15 min of sleep onset on the PSG can be used as part of the diagnostic criteria for diagnosing these disorders (see Table 5). An example of a hypnogram from a PSG in a patient with narcolepsy and SOREMP is shown in Fig. 3.

While the MSLT can be used in conjunction with PSG to assess for sleepiness and disorders of central hypersomnolence, a related but different test may be used to determine the ability to stay awake. The maintenance of wakefulness test (MWT) is a validated objective measure of the ability to stay awake for a defined period of time. Its main use is in occupations such as transportation industries and in pharmaceutical drug trials in the assessment of narcolepsy drugs. Unlike the MSLT, it cannot be used as a measure of sleepiness or as a 
Table 5 Comparison of common disorders of central hypersomnolence

\begin{tabular}{|c|c|c|}
\hline Narcolepsy type 1 & Narcolepsy type 2 & Idiopathic hypersomnia \\
\hline $\begin{array}{l}\text { Criteria A and B must be met: } \\
\text { A. Daily periods of irrepressible need } \\
\text { to sleep for } \geq 3 \text { months } \\
\text { B. Presence of one or both: } \\
\text { a. Cataplexy with a mean sleep } \\
\text { latency of } \leq 8 \text { min and } \geq 2 \text { SOREM } \\
\text { on MSLT } \\
\text { i. SOREM within } 15 \text { min of PSG } \\
\text { preceding MSLT can be used as one } \\
\text { SOREM } \\
\text { b. CSF hypocretin- } 1 \text { concentration is } \\
\leq 110 \text { pg/mL or }<1 / 3 \text { mean value of } \\
\text { normal subjects }\end{array}$ & $\begin{array}{l}\text { Criteria A-E must be met: } \\
\text { A. Daily periods of irrepressible need to sleep for } \\
\geq 3 \text { months } \\
\text { B. Mean sleep latency of } \leq 8 \text { min and } \geq 2 \text { SOREM } \\
\text { on MSLT } \\
\text { a. SOREM within } 15 \mathrm{~min} \text { of PSG preceding } \\
\text { MSLT can be used as one SOREM } \\
\text { C. Cataplexy is absent } \\
\text { D. Either CSF hypocretin- } 1 \text { has not been measured, } \\
\text { or levels are }>110 \mathrm{pg} / \mathrm{mL} \text { or }>1 / 3 \text { mean value } \\
\text { of normal subjects } \\
\text { E. Symptoms and MSLT findings are not better } \\
\text { explained by other causes }\end{array}$ & $\begin{array}{l}\text { Criteria A-F must be met: } \\
\text { A. Daily periods of irrepressible need to sleep for } \\
\geq 3 \text { months } \\
\text { B. MSLT shows }<2 \text { SOREM } \\
\text { a. SOREM within } 15 \text { min of PSG preceding MSLT can } \\
\text { be used as one SOREM } \\
\text { C. Cataplexy is absent } \\
\text { D. MSLT shows a mean sleep latency } \leq 8 \text { min, or the } \\
\text { total sleep time is } \geq 660 \text { min on a } 24-\text { PSG (not } \\
\text { typically done in the USA) } \\
\text { E. Insufficient sleep syndrome is ruled out } \\
\text { F. Symptoms and MSLT findings are not better } \\
\text { explained by other causes }\end{array}$ \\
\hline
\end{tabular}

ISCD-3 criteria for narcolepsy and idiopathic hypersomnia

SOREM sleep-onset rapid eye movement, MSLT multiple sleep latency test, $P S G$ polysomnogram, $C S F$ cerebrospinal fluid

diagnostic tool in the assessment of narcolepsy. A full discussion of these tests is beyond the scope of this article; however, it is important to note that prior studies have shown that the correlation between these tests is low [15], suggesting that the ability to stay awake and fall asleep is likely driven by independent processes.

\section{Evaluation of Movement Disorders of Sleep}

Movement disorders during sleep are seen frequently in the population, with one of the most common findings on PSG being periodic limb movements during sleep (PLMS). PLMS are short, involuntary movements occurring repetitively at about 20-40-s intervals. Clinically, they usually consist of big toe extension and dorsiflexion of the ankle, with occasional flexion at the knee and hip [16]. Though PLMS are common, their clinical significance remains controversial and they rarely cause sleepiness in isolation [17]. One major importance of reporting the periodic limb movement of sleep index (PLMSI) on a PSG is to alert clinicians to screen for the possibility of other sleep disorders or medical conditions which may be interfering with an individual's sleep. For example, an abnormally high PLMS index (defined as $>15 / \mathrm{h}$ in adults and $>5 / \mathrm{h}$ in children) can be seen in the setting of restless legs syndrome (RLS), certain medications (such as anti- depressants and anti-histamine medications), neurologic disorders (including peripheral neuropathy, spinal cord disease, narcolepsy, and multiple sclerosis), or medical conditions (including chronic kidney disease, uremia, and pregnancy). Untreated sleep-disordered breathing can also be associated with limb movements, though these movements are not typically incorporated in the PLMS index based on AASM scoring criteria. Restless legs syndrome may be of particular interest to clinicians as it is a clinical diagnosis that can be highly prevalent in certain populations, which can be supported by a high PLMS index (PLMSI) on a PSG [6]. Separately, periodic limb movement disorder (PLMD) is a relatively uncommon condition and requires PLMSI greater than 15 per hour in adults in the absence of other sleep conditions in which PLMS commonly occur such as untreated OSA, RLS, narcolepsy, or REM sleep behavior disorder [6]. Figure 4 provides an illustration of a patient with periodic limb movements during her sleep, without any other associated sleep disorder.

\section{Evaluation of Parasomnias}

Parasomnias are a group of sleep disorders that involve unwanted events or experiences that occur while falling asleep, during sleep, or waking up. These experiences may include abnormal movements, behaviors, emotions, perceptions, or

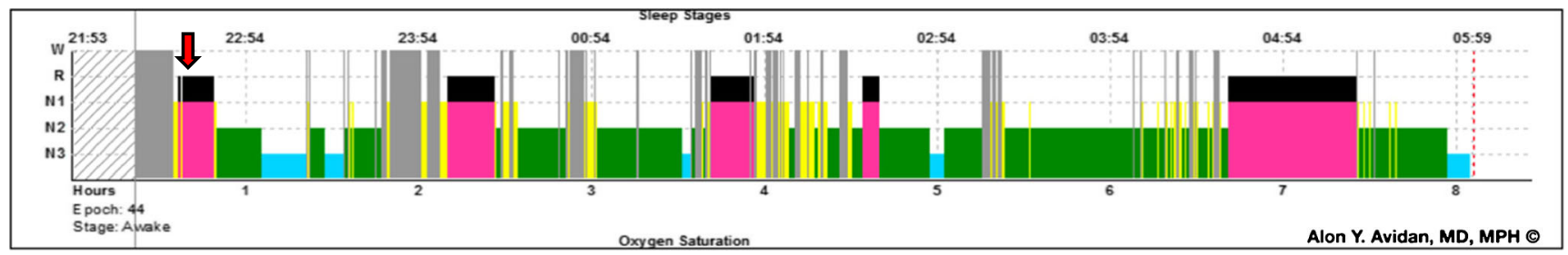

Fig. 3 Example of PSG hypnogram in narcolepsy. Hypnogram of nocturnal sleep with an early SOREMP (demarcated by the red arrow) and sleep fragmentation in a patient with narcolepsy. The colors of the bars represent the different sleep stages (stage W: gray-colored bar; stage

R: black/pink-colored bar; stage N1: yellow-colored bar; stage N2: greencolored bar; stage N3: blue-colored bar). Courtesy of Dr. Alon Y. Avidan, MD, MPH @. SOREMP, sleep-onset REM period 


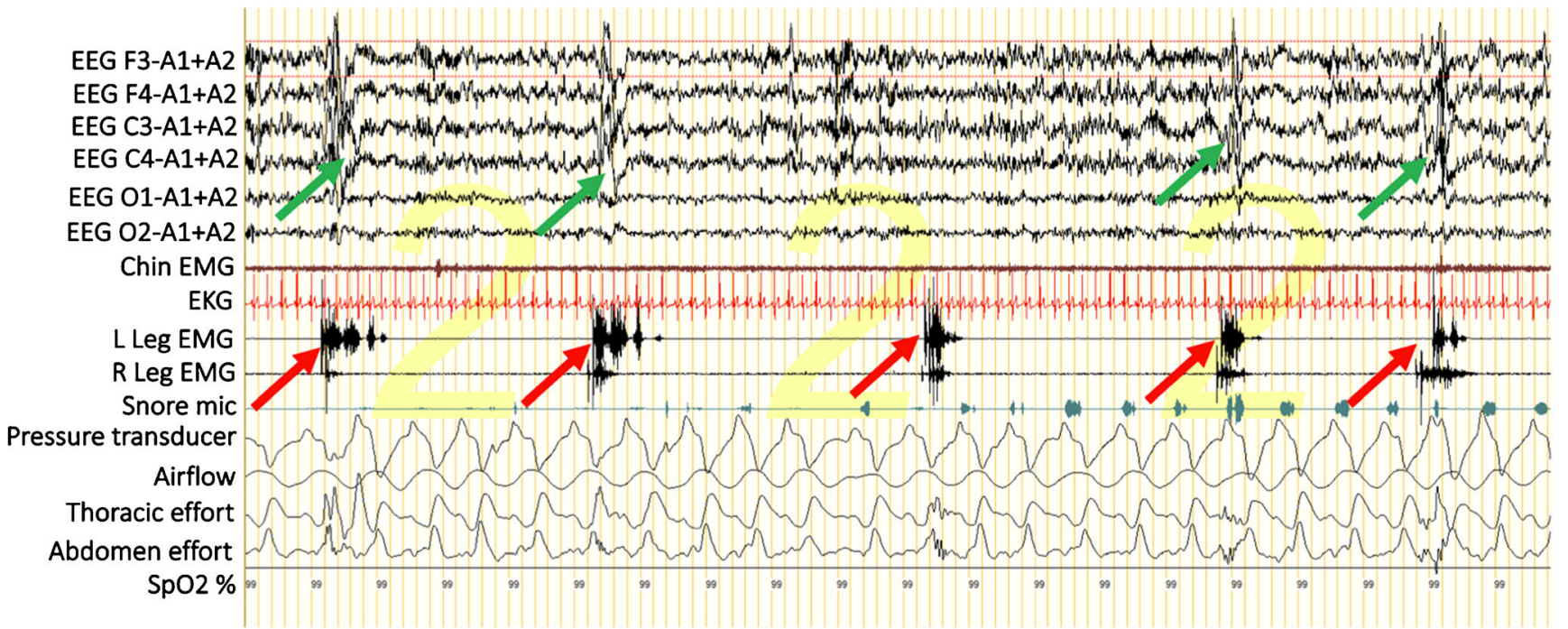

Fig. 4 Example of PSG in PLMD. PSG demonstrating periodic limb movements during sleep (PLMS), without any associated sleepdisordered breathing. Each limb movement (LM, indicated with red arrows) is $3-5 \mathrm{~s}$ in duration, has an amplitude of $20-60 \mu \mathrm{V}$ above the resting EMG amplitude, and is separated from the next consecutive LM by $10-20 \mathrm{~s}$. The LM together are part of a PLM series, which is defined as at least 4 consecutive LM, per the AASM scoring criteria. Limb movements are associated with EEG arousals (indicated by green arrows). These PSG epochs included the following channels: EEG F3$\mathrm{A} 1+\mathrm{A} 2$ left frontal in referential montage, EEG F4-A1+A2 right frontal in referential montage, EEG $\mathrm{C} 3-\mathrm{A} 1+\mathrm{A} 2$ left central in referential

dreams and can range from simple behavior to more complex interactions. Parasomnias are further divided into two categories, non-REM parasomnias or REM parasomnias, based on the stage of sleep from which they emerge.

\section{Non-REM Parasomnias}

Non-REM (NREM) parasomnias are events occurring out of NREM sleep and are more common in childhood and adolescents. Representative NREM parasomnias include confusional arousals, sleep terrors, somnambulism, sleep-related eating disorder, and sexsomnia. The occurrence of NREM parasomnias in adulthood is often related to sleep deprivation, the use of certain CNS-acting medications (hypnotics and anti-depressants), stress, and untreated sleep apnea due to its facilitation of sleep fragmentation of stage N3 sleep [6]. Events include impaired cognition during the episode and prolongation of sleep inertia (defined as prolonged difficulty waking up with a repeated return to sleep), with amnesia to the event, and can involve distressing elements such as crying, yelling, or injurious behavior. These disorders are generally able to be diagnosed on history alone; however, a PSG can be helpful if an episode is captured as they usually arise out of stage N3 sleep. It is important to note that a PSG is not required for these clinical diagnoses but can serve an important purpose to evaluate for other causes of sleep disruption which may trigger NREM parasomnia. For example, if there is a high montage, EEG C4-A1+A2 right central in referential montage, EEG $\mathrm{O} 1-\mathrm{A} 1+\mathrm{A} 2$ left occipital in referential montage, and EEG O2-A1+A2 right occipital in referential montage. Chin EMG, chin electromyography; EKG, electrocardiogram; L and R leg EMG, bilateral anterior tibialis electromyography; snore mic, snoring sensor/ microphone; pressure transducer, nasal airflow; airflow, oronasal thermal airflow sensor; thoracic effort, thoracic/chest effort channel; abdomen effort, abdominal effort channel; $\mathrm{SpO}_{2} \%$, oxygen saturation; PLMS, periodic limb movements during sleep; LM, limb movement; PLM, periodic limb movement; AASM, American Academy of Sleep Medicine

clinical suspicion for another sleep disorder, such as OSA or PLMD, along with NREM parasomnia, a PSG may be considered to evaluate for these other conditions as an underlying and treatable cause of the NREM parasomnia.

\section{Evaluation of REM Sleep Behavior Disorder}

Rapid eye movement (REM) sleep behavior disorder (RBD) is a parasomnia characterized by episodes of dream enactment behavior associated with augmentation of EMG tone during REM sleep, in the setting of a normal EEG and absence of other factors that can facilitate dream enactment such as OSA. These episodes themselves are often significantly distressing for patients and can cause potential injury, to both patients and bed partners.

RBD can serve as an important prodromal biomarker of neurodegenerative diseases such as Parkinson's disease and other synucleinopathies, such as Lewy body dementia (LBD) and multiple system atrophy (MSA) [18]. Video PSG with expanded EMG montage (arm and leg EMG leads) is an essential requirement in the diagnosis of RBD (ICSD-3, 2014). RBD requires the presence of REM sleep without atonia (RSWA) and dream enactment behavior. In fact, RBD is the only parasomnia in which a PSG is required to make an official diagnosis, as documentation of RSWA is needed, coupled with videographic evidence of dream enactment or a supportive clinical history. The following are official diagnostic criteria for RBD as provided by the AASM [14]: 
- Repeated episodes of sleep-related vocalization and/or complex motor behaviors

- Behaviors are documented by PSG to occur during REM sleep or based on the clinical history of dream enactment and are presumed to occur during REM sleep

- Presence of REM sleep without atonia on PSG

- Absence of epileptiform activity during REM sleep, unless RBD can be clearly distinguished from any concurrent REM sleep-related seizure disorder

- The sleep disturbance is not better explained by another sleep or medical disorder

An example of a patent with RSWA is shown in Fig. 5. In addition to evaluating for RBD with the above criteria, PSG is also helpful to assess for dream enactment behavior unrelated to RBD, such as episodes triggered by cortical arousals related to suboptimally controlled sleep apnea, a termed coined "pseudo-RBD.” [17].

\section{Evaluation of Nocturnal Seizure}

Sleep-related epilepsies typically begin in late childhood or adolescence and are characterized by short (usually $<1 \mathrm{~min}$ ) stereotyped movements that are often accompanied by arousals, vocalizations, frightened expression, complex behavior (such as bicycle pedaling and ballistic or dystonic limb movements), and a post-ictal state [17]. Nocturnal seizures typically arise out of NREM sleep; however, distinguishing them from other NREM phenomena, such as parasomnias, can prove challenging based on clinical history alone. For suspected nocturnal seizures, PSG can be helpful and supportive in confirming a suspected epilepsy diagnosis but can be equally important in ruling out other sleep disorders which may mimic a seizure. A PSG with expanded EEG montage is most helpful in this situation as epileptiform discharges and seizures typically arise in 30-60 min after sleep onset, in stages N2 and N3 sleep, and are most likely to be observed

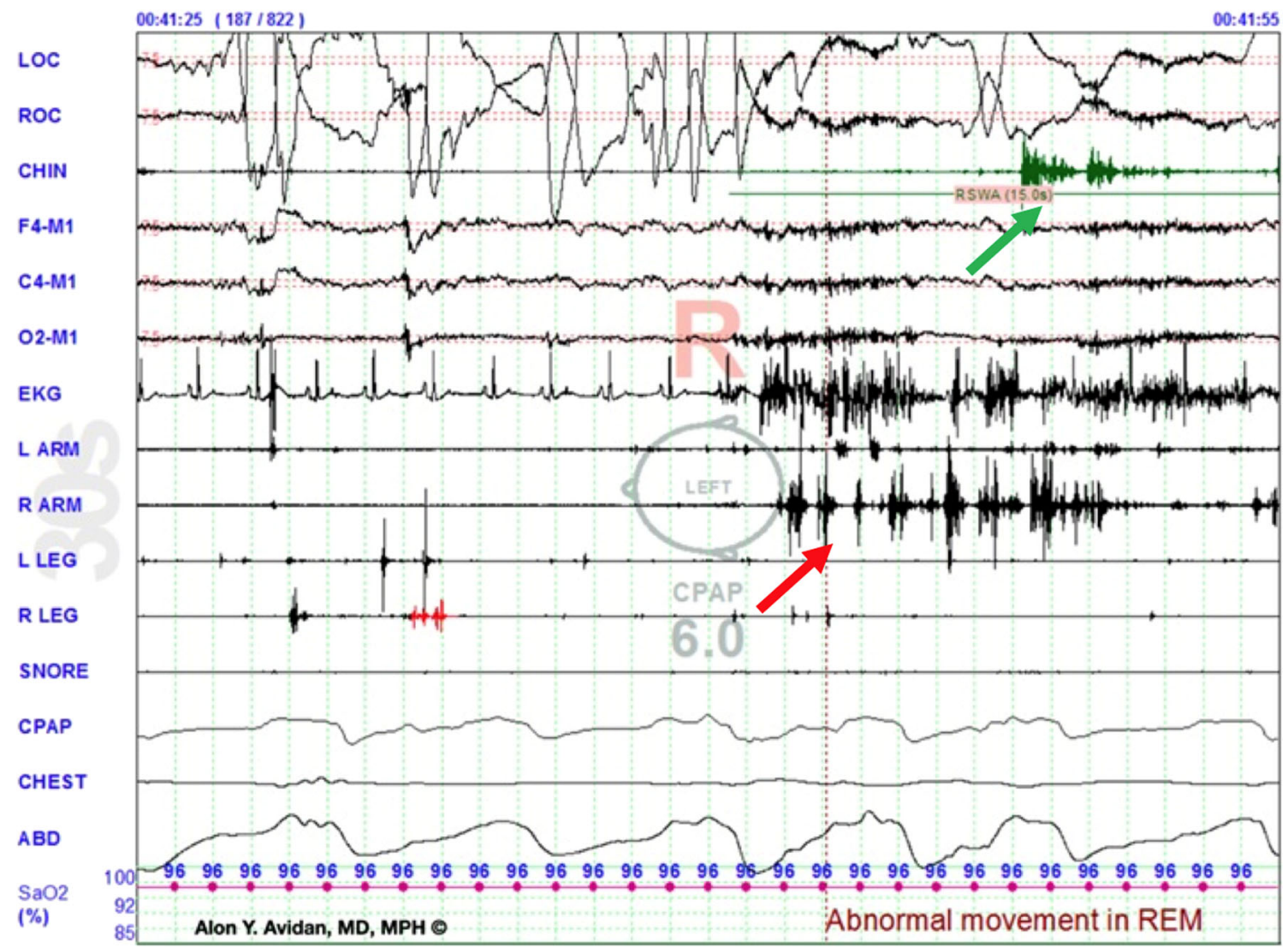

Fig. 5 Example of abnormal movement in REM as seen in greatest in right arm EMG (red arrow) and chin EMG (green arrow) in a 30-s epoch as evidence of RSWA which can be seen in RBD. Courtesy of Dr. Alon Y. Avidan, MD, MPH (C). The PSG epoch included the following channels: LOC and ROC, left and right electrooculogram; chin EMG, chin electromyography; F4-M1, EEG channel right frontal in reference to the contralateral mastoid process; C4-M1, EEG channel right central in reference to the contralateral mastoid process; O2-M1, EEG channel right occipital in reference to the contralateral mastoid process; EKG, electrocardiogram; L arm and $\mathrm{R}$ arm, left arm and right arm electromyography; L leg and $\mathrm{R} \mathrm{leg}$, left leg and right leg electromyography; snore, snore sensor/microphone; CPAP, CPAP airflow channel pressure set at $6.0 \mathrm{~cm} \mathrm{H}_{2} \mathrm{O}$ here; chest, thoracic/chest effort channel; Abd, abdominal effort channel; $\mathrm{SaO}_{2}(\%)$ or $\mathrm{SpO}_{2} \%$, oxygen saturation; RSWA, REM sleep without atonia; RBD, REM behavior disorder; EMG, electromyography 
with expanded EEG channels. However, even with the most sensitive study parameters, the yield of capturing an epileptiform discharge is $<50 \%$ [19], and thus, PSG can be helpful but is not required to make the clinical diagnosis of nocturnal seizure. We include here an example of a patient with a nocturnal seizure on PSG seen maximal at frontotemporal regions in Fig. 6.

\section{Limitations and Challenges of Polysomnography}

It is important to note the limitations of PSG in both interpretation and diagnosis. Many sleep disorders have night-to-night variability; thus, a single night snapshot may not truly reflect an individual's normal sleep pattern. In addition, there can be a "first-night" effect, with limited sleep time and worsened sleep quality on the first night of sleep in unfamiliar surroundings [2]. If there are lower levels of deeper stages of sleep, it may reflect this first night effect and thus may not accurately portray an individual's sleep disorder. Home sleep studies have the benefit of being performed in a patient's home environment; however, they do not have the advantage of having a sleep technologist who corrects potential technical issues that may arise in the middle of the night [20]. Self-titration positive airway pressure (auto PAP devices) has improved some care delivery as well, though it cannot be used if the patient has central sleep apnea (CSA), hypoventilation syndromes, congestive heart failure, or chronic obstructive pulmonary disease $[7,21]$. Practice guidelines through the AASM help with the standardization of sleep labs [22•]. However, there are still practice variations in both the technical and interpretation differences inherent to the individualized practice.

Home sleep testing (see Fig. 7) which traditionally measures airflow, pulse oximetry, and respiratory effort has become an acceptable technology, with clinical trials showing comparable diagnosis and treatment initiation among patients with a high likelihood of OSA [23, 24]. There have been multiple comparative studies between PSG and HSAT, which have shown similar diagnosis and successful treatment rates with CPAP, when excluding patients with cardiovascular disease and other medical comorbidities, and in those with a high likelihood of having OSA $[25,26]$. This is an important point in that HSAT should not be used to "rule out" OSA, especially if results are negative in a patient with a low pretest probability of having SRBD [24]. This is one of the biggest limitations of

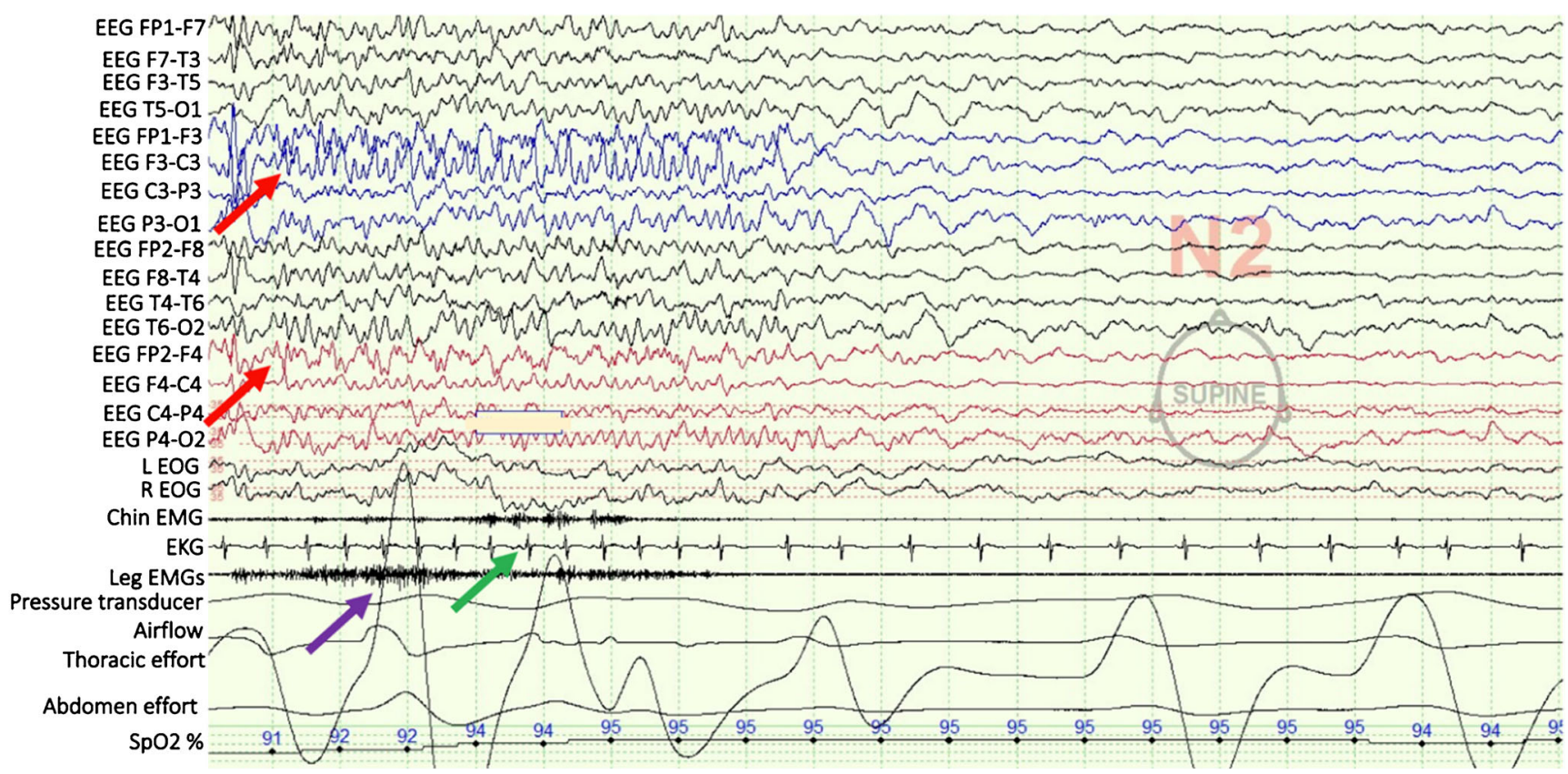

Fig. 6 Example of PSG in nocturnal frontal lobe epilepsy. Nocturnal PSG in a 20-s epoch during N2 sleep demonstrates the emergence of ictal scalp EEG discharges over a period of $10 \mathrm{~s}$. EEG fast activity during a seizure is seen maximal at the bilateral frontotemporal regions (red arrows), disrupting the background. The EKG indicates ictal tachycardia (green arrow) consistent with ictal autonomic hyperactivity synchronous with seizure. There are leg movements present in bilateral anterior tibialis EMG (purple arrow) during the recorded seizure. There is demonstrated oxygen desaturation at the ictal onset. The EOG leads indicate no rapid eye movements, and respiratory channels do not demonstrate tachypnea or change in the respiratory effort during the event. This 20-s PSG epoch included the following channels: longitudinal bipolar extended EEG montage in the international 10-20 system including prefrontal $(\mathrm{FP})$, frontal $(\mathrm{F})$, temporal $(\mathrm{T})$, parietal $(\mathrm{P})$, and occipital $(\mathrm{O})$ electrodes with odd numbers indicating left hemisphere and even numbers indicating right hemisphere. L EOG and R EOG, left and right electrooculogram; chin EMG, chin electromyography; EKG, electrocardiogram; leg EMGs, leg electromyography; pressure transducer, nasal airflow; airflow, oronasal thermal airflow sensor; thoracic effort, thoracic/chest effort channel; abdomen effort, abdominal effort channel; $\mathrm{SpO}_{2} \%$, oxygen saturation 
portable testing [27]. Another disadvantage of home testing is the lack of an EEG parameter, which precludes the assessment of cortical arousals and, indirectly, respiratory effort-related arousals (RERAs). As there is a subset of patients in whom respiratory events are associated with arousal rather than desaturations, HSAT will not capture this subgroup of patients in diagnosing OSA [12]. The benefit of cost and convenience with home sleep testing may outweigh these disadvantages; however, the accuracy and quality of portable testing remain to be studied and newer developments in technology may aid accuracy (Table 6).

\section{Trends and Developments}

An interesting approach in recent years includes a movement towards precision medicine, which aims to predict and individualize health care using different data sources that tailor to personal health concerns [28•]. Extraction of physiologic metrics from PSG can be utilized to evaluate the impact of a treatment or behavioral intervention on an individual's sleep. One way this approach may help in the classification and personalization of diagnosis is a better characterization of subtypes of disorders such as OSA. OSA is a clinically heterogeneous disorder with several subtypes that may respond differently to various interventions [29]. PSG can further delineate these variables, such as the arousal index, apnea duration, and degree of hypoxemia, to better characterize which treatment modalities (including CPAP, oral appliance, sleep surgery, or pharmacotherapy) may be most beneficial in specific groups of patients [28].

There is also significant information gained from both traditional PSG and home sleep tests which we do not currently apply to clinical decisions. This includes data about normal sleep processes, age-related changes, and gender differences. As we continue to learn more about the fundamental neuroscience of sleep functions, we can apply the information gathered from PSG to better understand the evolution of other neurologic and medical conditions. Applying these sleep variables to the study of neurodegenerative conditions, in particular, has become an important recent trend to better understand memory disorders. For example, a recent study evaluated asymptomatic older adults for SRBD with HSAT. A large majority of patients $(75 \%)$ met the criteria for SRBD based on AHI $>15$ alone. Interestingly, there was no difference between these groups in measures of subjective sleep quality, daytime sleepiness, or cognitive performance. Advanced neuroimaging magnetic resonance imaging (MRI) and (positron emission tomography) PET voxel-wise comparison in this study revealed that untreated SRBD presented greater amyloid

Table 6 Comparison between the HSAT and PSG elements

\begin{tabular}{|c|c|c|}
\hline & HSAT & PSG \\
\hline Respiratory channels & Measured, including $\mathrm{O}_{2}$ sensor & Measured, including $\mathrm{O}_{2}$ sensor \\
\hline EEG channels/sleep staging & Not measured & Measured \\
\hline EMG channels/limb movement & Not measured & Measured \\
\hline EOG channels & Not measured & Measured \\
\hline ECG channel & Not measured & Measured with single-lead ECG \\
\hline Video monitoring & Not measured & Measured \\
\hline Patient position information & Measured on most devices & Measured \\
\hline Low-to-moderate pretest probability of OSA & $\begin{array}{l}\text { Can underestimate the severity of } \\
\text { OSA in mild cases }\end{array}$ & Provides more accurate diagnosis of milder cases \\
\hline Moderate-to-high pretest probability of OSA & $\begin{array}{l}\text { Accuracy may be comparable to } \\
\text { PSG }\end{array}$ & Accuracy is comparable to HSAT \\
\hline $\begin{array}{l}\text { Evaluation for conditions such as narcolepsy, } \\
\text { parasomnias, RBD, SRE, and PLMD }\end{array}$ & Not recommend & Recommended \\
\hline $\begin{array}{l}\text { Use in patients with cardiopulmonary comorbidities } \\
\text { or concern for hypoventilation }\end{array}$ & Not recommend & Recommended \\
\hline $\begin{array}{l}\text { Assessment of initial response to weight loss, oral } \\
\text { appliance therapy, or surgery for OSA }\end{array}$ & Not recommended & Recommended \\
\hline Technical limitations & $\begin{array}{l}\text { Higher technical failure rate with } \\
\text { varied sensor technology }\end{array}$ & $\begin{array}{l}\text { More reliable data with a trained technologist to correct } \\
\text { artifacts and troubleshoot technical issues }\end{array}$ \\
\hline Convenience & $\begin{array}{l}\text { Conducted at home, more } \\
\text { accessible }\end{array}$ & Conducted at the sleep laboratory, less accessible \\
\hline Cost & $\begin{array}{l}\text { Less expensive with lower labor } \\
\text { cost }\end{array}$ & More expensive with higher labor cost \\
\hline
\end{tabular}

$H S A T$ home sleep apnea test, $P S G$ polysomnography, $E E G$ electroencephalography, $E M G$ electromyography, $E O G$ electrooculogram, $E M G$ electromyography, ECG electrocardiography, OSA obstructive sleep apnea, REM rapid eye movement, SRE sleep-related epilepsy, PLMD periodic limb movement disorder 


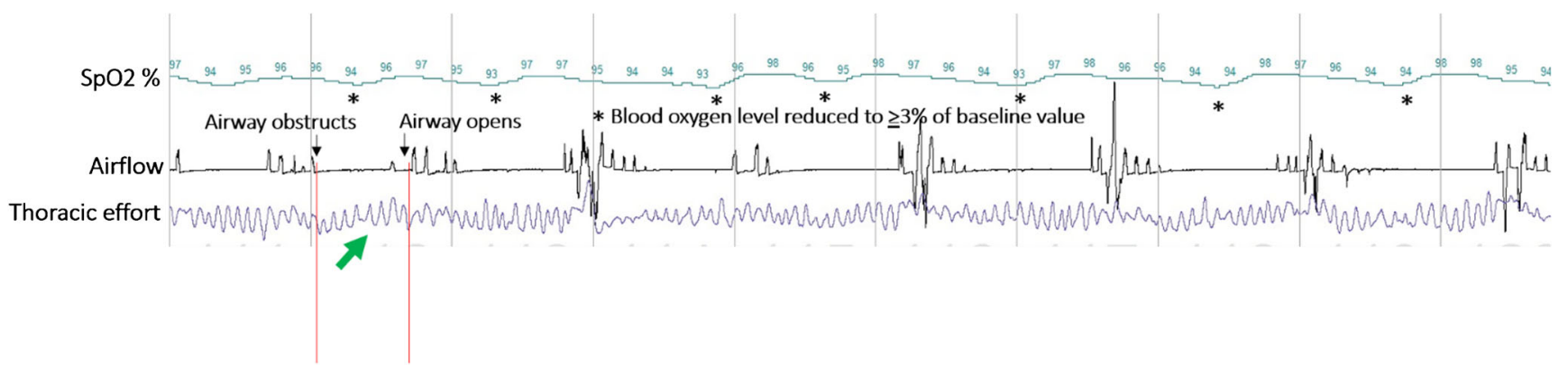

Fig. 7 Home sleep apnea test. A 5-min epoch from a home sleep apnea test which demonstrates severe obstructive sleep apnea. The respiratory channels demonstrate significant airflow limitation (example between

deposition and metabolism over brain regions (posterior cingulate cortex and precuneus areas) which are alternated in Alzheimer's disease [30 ${ }^{\bullet}$. Thus, screening for sleep disorders, such as OSA, may be beneficial in identifying asymptomatic older adults to enroll in other clinical trials aimed at preventing cognitive decline. This study provides additional new evidence beyond the current US Preventive Services Task Force (USPSTF) guidelines which conclude the currently insufficient evidence to assess the benefits and harms of screening for OSA in asymptomatic adults [31••]. Furthermore, recent Alzheimer's disease biomarker studies of patients with subjective cognitive impairment (SCI) but normal cognitive testing found greater amyloid deposition based on the cerebrospinal fluid analysis in patients with untreated OSA [32•]. Patients with untreated OSA had lower serum and cerebrospinal fluid amyloid levels compared to both controls and OSA patients treated with CPAP indicating PAP therapy as a possible early cognitive therapeutic strategy in patients with SCI and OSA [32•].

Developments in home sleep testing have gained momentum as well, in an effort to minimize the traditional limitations of portable testing. Toedebusch et al. explored a research protocol which combined singlechannel frontal EEG and EMG leads, with traditional respiratory parameters to monitor older cognitively normal or very mildly impaired adults, who may not be able to present for an in-lab test [33•]. They demonstrated a feasible option for older adults along with instructional video and support. Further expansion of HSAT includes FDA-approved disposable equipment to minimize handling and reuse of equipment. This has become especially important now to minimize potential SARSCoV-2 transmission and cross-contamination in the current 2019-2020 pandemic [34].

Within the last 5 years, we have also seen an expanding focus on wearable sleep tracker devices and consumer sleep technology. Though there is more data, there is a challenge in the validity and reliability of this data to be used clinically beyond the general wellness information. There has been an increasing number of vertical red bars), accompanied by cyclic desaturations (indicated by asterisks) with preserved respiratory effort (example green arrow), hallmarks of OSA

studies evaluating the performance of wearable sleep trackers against standard PSG; however, most were conducted in the laboratory and not under natural sleep settings [35]. Each device uses a proprietary algorithm and when compared to PSG has variability in measures of sleep/wake. Importantly, only a few commercial devices (i.e., Dreem $2^{\mathrm{TM}}$ ) used EEG to measure staging as is standard with PSG [36]. These newer devices using either headband or around the ear dry electrodes for EEG are currently in validations studies, and the hope will be to gain more sleep information longitudinally [37]. So far, early validation studies have shown feasible and accessible ways to acquire sleep data in newer montages and a moderate range of agreement on the group level for all sleep staging combined based on blinded rated AASM scoring [38].

Newer smartphone devices are also equipped with building accelerometers which can interface wirelessly with more personalized data. Some studies reveal participants reported improved sleep through changes in sleep habits [39]. These devices enhance awareness of sleep quality and sleep hygiene, which may indirectly impact sleep through a "placebo-like" mechanism. Mobile applications will continue to evolve, and further wireless technology innovations will continue to push forward the expansion of the traditional polysomnogram.

\section{Conclusion}

Polysomnography has been a longstanding fundamental diagnostic test and continues to hold the cornerstone role in diagnosing many sleep disorders. Evolving technologies, including precision medicine and the development of advanced outof-lab testing modalities, continue to grow and have emerged in certain populations to be a clinically beneficial and more convenient diagnostic tool. There remain many patient populations who are still best served with a PSG. Future research is needed to determine how to best apply PSG to certain populations and when to consider alternative testing options. 


\section{Compliance with Ethical Standards}

Conflict of Interest The authors declare that they have no conflict of interest.

\section{References}

Papers of particular interest, published recently, have been highlighted as:

- Of importance

•- Of major importance

1. Hirshkowitz M. Polysomnography challenges. Sleep Med Clin. 2016;11(4):403-11.

2. Simons PJ, Overeem S. Polysomnography: recording, analysis and interpretation. Sleep Disord Neurol Pract Approach. 2018;17:1329.

3. Loddo G, Lopez R, Cilea R, Dauvilliers Y, Provini F. Disorders of arousal in adults: new diagnostic tools for clinical practice. Sleep Sci Pract. 2019;3(1):5.

4. Kayabekir M. Sleep physiology and polysomnogram, physiopathology and symptomatology in sleep medicine. Clin Neurol. 2019. IntechOpen.

5. Patil SP. What every clinician should know about polysomnography. Respir Care. 2010;55(9):1179-95.

6. Berry RB, Wagner MH. Sleep medicine pearls book. Elsevier Health Sciences; 2014.

7.• Kapur VK, Auckley DH, Chowdhuri S, Kuhlmann DC, Mehra R, Ramar K, et al. Clinical practice guideline for diagnostic testing for adult obstructive sleep apnea: an American Academy of Sleep Medicine clinical practice guideline. J Clin Sleep Med. 2017;13(03):479-504 Most recent AASM Clinical Practice Guidelines on the diagnostic testing of OSA.

8. Kim H, Young T. Subjective daytime sleepiness: dimensions and correlates in the general population. Sleep. 2005;28(5):625-34.

9. Osman AM, Carter SG, Carberry JC, Eckert DJ. Obstructive sleep apnea: current perspectives. Nat Sci Sleep. 2018;10:21-34.

10. Chung F, Yegneswaran B, Liao P, Chung SA, Vairavanathan S, Islam S, et al. STOP questionnaire: a tool to screen patients for obstructive sleep apnea. Anesthesiology. 2008;108(5):812-21.

11. Morales CR, Hurley S, Wick LC, Staley B, Pack FM, Gooneratne NS, et al. In-home, self-assembled sleep studies are useful in diagnosing sleep apnea in the elderly. Sleep. 2012;35(11):1491-501.

12. Collop N. Home sleep testing: appropriate screening is the key. Editorial commentary on Morales et al. In-home, self-assembled sleep studies are useful in diagnosing sleep apnea in the elderly. Sleep. 2012;35:1491-501.

13. Kendzerska T, Gershon AS, Hawker G, Leung RS, Tomlinson G. Obstructive sleep apnea and risk of cardiovascular events and allcause mortality: a decade-long historical cohort study. PLoS Med. 2014;11(2).

14. The international classification of sleep disorders. Westchester, IL: American Academy of Sleep Medicine; 2014.

15. Sangal RB, Thomas L, Mitler MM. Maintenance of wakefulness test and multiple sleep latency test. Measurement of different abilities in patients with sleep disorders. Chest. 1992;101:898-902.

16. Bara-Jimenez W, Aksu M, Graham B, Sato S, Hallett M. Periodic limb movements in sleep: state-dependent excitability of the spinal flexor reflex. Neurology. 2020;54:1609-16.

17. Silber MH. Diagnostic approach and investigation in sleep medicine. CONTINUUM: Lifelong Learn Neurol. 2017;23(4):973-88.
18. Postuma RB. Diagnosing REM sleep behavior disorder in Parkinson's disease - can we avoid the polysomnogram? Mov Disord. 2014;29(6):713-4.

19. Bisulli F, Vignatelli L, Provini F, Leta C, Lugaresi E, Tinuper P. Parasomnias and nocturnal frontal lobe epilepsy (NFLE): lights and shadows - controversial points in the differential diagnosis. Sleep Med. 2011;12(2):S27-32.

20. Mendonca F, Mostafa SS, Morgado-Dias F, Ravelo-Garcia AG, Penzel T. A review of approaches for sleep quality analysis. IEEE Access. 2019;7:24527-46.

21. Rosen CL, Auckley D, Benca R, Foldvary-Schaefer N, Iber C, Kapur V, et al. A multisite randomized trial of portable sleep studies and positive airway pressure autotitration versus laboratory-based polysomnography for the diagnosis and treatment of obstructive sleep apnea: the HomePAP study. Sleep. 2012;35(6):757-67.

22. Berry RB, Brooks R, Gamaldo CE. The AASM manual for the scoring of sleep and associated events: rules, terminology and technical specifications, version 2.6. 0. American Academy of Sleep Medicine, Darien, Illinois; 2020. Most recent scoring manual from the American Academy of Sleep Medicine (AASM).

23. Atwood CW Jr. "The times they are a changin:" home diagnosis of sleep apnea has arrived. Sleep. 2012;35(6):735-6 Commentary on Rosen et al. A multisite randomized trial of portable sleep studies and positive airway pressure auto titration versus laboratory-based polysomnography for the diagnosis and treatment of obstructive sleep apnea: the HomePAP study. Sleep. 2012 Jun 1;35(6):757-67.

24. Cairns A, Poulos G, Bogan R. Who is getting tested for obstructive sleep apnea using a portable recording system? Test results from 193,221 patients. J Clin Sleep Med. 2014;10(11):1193-8.

25. Santos-Silva R, Sartori DE, Truksinas V, Truksinas E, Alonso FF, Tufik S, et al. Validation of a portable monitoring system for the diagnosis of obstructive sleep apnea syndrome. Sleep. 2009;32(5): 629-36.

26. de Oliveira AC, Martinez D, Vasconcelos LF, Gonçalves SC, do Carmo Lenz M, Fuchs SC, et al. Diagnosis of obstructive sleep apnea syndrome and its outcomes with home portable monitoring. Chest. 2009;135(2):330-6.

27. Donovan LM, Patel SR. Making the most of simplified sleep apnea testing. Ann Intern Med. 2017;166(5):366-7.

28. Lim DC, Mazzotti DR, Sutherland K, Mindel JW, Kim J, Cistulli $\mathrm{PA}$, et al. Reinventing polysomnography in the age of precision medicine. Sleep Med Rev. 2020;101313 Recent article which suggests how PSG can be used in the era of precision medicine, to determine more individualized approaches and solutions towards the treatment of sleep disorders.

29. Eckert DJ, White DP, Jordan AS, Malhotra A, Wellman A. Defining phenotypic causes of obstructive sleep apnea. Identification of novel therapeutic targets. Am J Respir Crit Care Med. 2013;188(8):996-1004.

30. André C, Rehel S, Kuhn E, Landeau B, Moulinet I, Touron E, et al. Association of sleep-disordered breathing with Alzheimer disease biomarkers in community-dwelling older adults: a secondary analysis of a randomized clinical trial. JAMA Neurol. 2020; Supportive evidence suggesting there may be greater amounts of beta-amyloid deposition in untreated OSA, a finding which may be helpful in the treatment and prevention of certain neurodegenerative conditions (such as Alzheimer's dementia).

31.• Bibbins-Domingo K, Grossman DC, Curry SJ, Davidson KW, Epling JW, Garcia FA, et al. Screening for obstructive sleep apnea in adults: US Preventive Services Task Force recommendation statement. JAMA. 2017;317(4):407-14 Most recent US Preventive Services Task Force recommendation on the screening for OSA.

32. Liguori C, Mercuri NB, Izzi F, Romigi A, Cordella A, Sancesario $\mathrm{G}$, et al. Obstructive sleep apnea is associated with early but possibly modifiable Alzheimer's disease biomarkers changes. Sleep. 
2017;40(5):Zsx011 Further supportive evidence suggesting an association between beta-amyloid deposition in untreated OSA, a finding which may be helpful in the treatment and prevention of certain neurodegenerative conditions (such as Alzheimer's dementia).

33. Toedebusch CD, McLeland JS, Schaibley CM, Banks IR, Boyd J, Morris JC, et al. Multi-modal home sleep monitoring in older adults. JoVE (J Vis Exp). 2019;26(143):e58823. A review of enhancing portable sleep testing in older adults with additional modalities.

34. Quintos A, Tavella R, Doghramji K. Disposable equipment for efficient home sleep apnea testing and minimizing human handling at the sleep laboratory. Thomas Jefferson University Digital Commons 2020 House Staff Quality Improvement and Patient Safety Conference.

35. De Zambotte M, Cellini N, Goldstone A, Colrain IM, Baker FC. Wearable sleep technology in clinical and research settings. Med Sci Sports Exerc. 2019;51(7):1538-57.
36. Roomkham S, Lovell D, Cheung J, Perrin D. Promises and challenges in the use of consumer-grade devices for sleep monitoring. IEEE Rev Biomed Eng. 2018;11:53-67.

37. Arnal PJ, Thorey V, Ballard ME, Hernandez AB, Guillot A, Jourde $\mathrm{H}$, et al. The Dreem headband as an alternative to polysomnography for EEG signal acquisition and sleep staging. bioRxiv. 2019;1: 662734.

38. Sterr A, Ebajemito JK, Mikkelsen KB, Bonmati-Carrion MA, Santhi N, della Monica C, et al. Sleep EEG derived from behindthe-ear electrodes (cEEGrid) compared to standard polysomnography: a proof of concept study. Front Hum Neurosci. 2018;12:452.

39. Liang Z, Ploderer B. Sleep tracking in the real world: a qualitative study into barriers for improving sleep. In Proceedings of the 28th Australian Conference on Computer-Human Interaction. 2016 (pp. 537-541).

Publisher's Note Springer Nature remains neutral with regard to jurisdictional claims in published maps and institutional affiliations. 